\title{
A simplified and modified procedure to culture brain glioma stem cells from clinical specimens
}

\author{
BO QIU, DONGYONG ZHANG, JUN TAO, ANHUA WU and YUNJIE WANG \\ Department of Neurosurgery, First Hospital of China Medical University, Shenyang, Liaoning 110001, P.R. China
}

Received July 1, 2011; Accepted September 26, 2011

DOI: $10.3892 / \mathrm{ol} .2011 .433$

\begin{abstract}
In recent years, the theory of brain glioma stem cells (BGSCs) has facilitated the study of gliomas. BGSCs have been accepted as the origin of gliomas and determine their biological features. Numerous efforts have been made to probe into the biological characteristics and behaviors of BGSCs. However, the culturing of target cells remains the essential first step for research on BGSCs. In this study, we established a simplified procedure to culture and isolate BGSCs from samples of clinical glioma patients. Samples of 17 glioma patients were included in the study, and the processed glioma cells were grown in serum-free stem cell media. After the tumor spheres appeared, a proliferation assay, a single-cellderived colonies formation assay and an induced differentiation assay were carried out, followed by an immunocytochemistry assay. Serial passage was used to purify the target cells, whereas neither animal experiments nor sorting techniques were included. As a result, CD133 ${ }^{+}$BGSCs from 8 out of 17 patients were grown and maintained in a serum-free condition combined with EGF, FGF and B-27 supplements. The tumor sphere cells were serially passaged and showed pluripotency in an induced differentiation assay. Immunocytochemistry identified the committed markers (CD133, GFAP and TU-20) and confirmed the cells were BGSCs and their progeny. The results proved that $\mathrm{CD} 133^{+}$BGSCs from resected glioma tissue may be cultured in serum-free stem cell media, and may also be purified by conditioned culture combining serial passage, which is time-saving and cost-effective, and allows the cells to be used for subsequent research. The cell sorting techniques and animal experiments of tumorigenecity are optional. Thus, this modified procedure is more practical and feasible than other available procedures.
\end{abstract}

Correspondence to: Dr Yunjie Wang, Department of Neurosurgery, First Hospital of China Medical University, No.155, North Nanjing Street, Heping, Shenyang, Liaoning 110001, P.R. China

E-mail:wyj024@vip.sina.com

Key words: glioma, brain glioma stem cells, CD133, proliferation, self-renewal

\section{Introduction}

As the most refractory tumors of the central nervous system, gliomas comprise 33.3-58.9\% of intracranial tumors. The highgrade gliomas in particular (e.g., glioblastoma multiforme) have a dismal prognosis and a high recurrence rate; despite advances in multimodality therapy, the median survival is only 1 year $(1,2)$. In recent years, the cancer stem cells theory (3) and the finding of glioma stem cells (4-6) have been the highlights of glioma research. Brain glioma stem cells (BGSCs) have been proven to be the 'seed' cells of, and be responsible for, the initiation and development of gliomas, and thus have become new targets of glioma therapy (7). Currently the research on BGSCs mainly focuses on cell experiments, which demand a large number of cultured BGSCs as the first step. In this study, we introduce a simplified and modified procedure to culture, identify and purify BGSCs from resected samples of glioma patients, which is much more convenient and practical for the subsequent BGSC experiments.

\section{Materials and methods}

Tissue collection and grading. Brain glioma specimens were obtained within $30 \mathrm{~min}$ of surgical resection and were processed. The pathological grade of each specimen was confirmed by chief neuropathologists according to World Health Organization criteria. All 17 cases were inpatients in the Department of Neurosurgery of the First Hospital of China Medical University, China, between October 2008 and January 2009. The protocol of this study was approved by the Institutional Review Boards of the First Clinical Hospital, China Medical University.

Reagents and antibodies. Fetal bovine serum (FBS, qualified, Gibco, Invitrogen, Carlsbad, CA, USA), Dulbecco's modified Eagle's medium (DMEM)/F12 (Gibco), B-27 supplement (without serum and vitamin A, Invitrogen), recombinant human epidermal growth factor (rhEGF, Invitrogen), basic recombinant human fibroblast growth factor (rhFGF-b, Invitrogen), mouse monoclonal antiCD133 antibodies (Abcam, Cambridge, UK), monoclonal rabbit anti-GFAP (glial fibrillary acidic protein) antibodies (Bioworld, Dublin, OH, USA), TU-20 (monoclonal mouse anti- $\beta$-tubulin III isoform, C-terminus, Millipore, Billerica, MA, USA), Cy3-conjugated goat anti-mouse secondary 
antibodies (Sigma, St. Louis, MA, USA), Cy3-conjugated goat anti-rabbit secondary antibodies (Sigma), fluorescein isothiocyanate (FITC)-conjugated goat anti-mouse antibodies (Sigma) and 4,6-diamidino-2-phenylindole (DAPI, Sigma) were used. The other common reagents were all analytically pure.

Culture of primary glioma cells and tumor spheres. Tumor-sphere cultures were performed according to reported procedures (4-6,8), with minor modifications. Following resection, the tumor tissues were washed, minced in phosphatebuffered saline (PBS) and subjected to enzymatic dissociation. The tissues were then mechanically dissociated with a graded series of fire-polished Pasteur pipettes, and passed through a series of cell strainers, and centrifuged at $800 \mathrm{x} \mathrm{g}$ for $5 \mathrm{~min}$. Tumor cells were resuspended in DMEM/F12 containing $15 \%$ FBS and plated at a density of $2 \times 10^{5}$ live cells $/ \mathrm{ml}$. When tumor cells grew as monolayers in flasks, the medium was changed to committed stem cell medium (serum-free DMEM/F-12, 1:50 of B-27 supplement, $20 \mathrm{ng} / \mathrm{ml} \mathrm{rhEGF}, 20 \mathrm{ng} / \mathrm{ml} \mathrm{rhFGF}-\mathrm{b}$, $100 \mathrm{IU} / \mathrm{ml}$ penicillin $\mathrm{G}$ and $100 \mu \mathrm{g} / \mathrm{ml}$ streptomycin). Fresh rhEGF and rhFGF-b were added each week and the medium was changed twice a week. Cells were maintained in a standard tissue culture incubator with $5 \% \mathrm{CO}_{2}$ and $100 \%$ relative humidity at $37^{\circ} \mathrm{C}$.

Proliferation assay and limited dilution assays. In the proliferation assay, when suspending cells emerged and the primary tumor spheres were clear, they were collected and dissociated into single-cell suspensions through a fire-polished Pasteur pipette, and then reseeded in the stem cell media at the same cell density. Each cell line was serially passaged over 4 generations. To evaluate the self-renewal capacity of tumor spheres, tumor spheres were mechanically dissociated into single-cell suspensions and reseeded into 96-well microwell plates by limited dilution at a cell density of 1-2 live cells per well. Each well was fed with fresh stem cell media every 3-4 days. The formation of cell clones was inspected under a phase-contrast microscope after 7-10 days.

Immunocytochemistry assays. The tumor spheres of approximately the 4 th passage were collected and plated onto anti-peeling slides and incubated with stem cell media for $4 \mathrm{~h}$. Following firm adhesion, the tumor spheres were fixed in $4 \%$ paraformaldehyde and incubated at $4{ }^{\circ} \mathrm{C}$ with mouse monoclonal anti-CD133 antibodies diluted at 1:200 according to the manufacture's instructions. Cy3-conjugated goat anti-mouse secondary antibodies diluted at 1:50 were added $24 \mathrm{~h}$ later and incubated for $2 \mathrm{~h}$ at room temperature.

To assess the multipotency of the tumor spheres, the tumor spheres were harvested and subjected to a differentiation assay by plating onto coverslips precoated with poly-L-lysine in DMEM/F-12 media containing 15\% FBS and in the absence of growth factors or B-27 supplement. The media were changed every 2 days. The differentiated cells were fixed 7 days later, incubated with monoclonal rabbit ant-GFAP (1:200) for glial cells and TU-20 (1:200) for neurons overnight at $4^{\circ} \mathrm{C}$. Secondary antibodies (Cy3-conjugated goat anti-rabbit and FITC-conjugated goat anti-mouse, 1:250) were then added and incubated for $2 \mathrm{~h}$ at room temperature.
For all of the staining, the cell nuclei were counterstained with DAPI for $5 \mathrm{~min}$. In the control samples, primary antibodies were replaced by isotype IgG. A fluorescence microscope (Olympus BX61) was then used to observe and capture images of the results. The excitation wavelength was $554 \mathrm{~nm}$ for Cy3, $488 \mathrm{~nm}$ for FITC and $350 \mathrm{~nm}$ for DAPI.

\section{Results}

Tumor spheres culture. Tumor spheres were successfully cultured in 8 out of all 17 cases of glioma samples, including 2 primary glioblastoma multiformes (WHO grade IV), 1 recurrent anaplastic oligodendroglioma and 5 anaplastic astrocytomas (WHO grade III). The patients consisted of 3 males and 5 females. The ages varied from 44-73 years old, and the average age was 53.75 years old.

The monolayers formed 24-72 $\mathrm{h}$ after the single-cell suspensions were seeded in the serum-containing media. When switched into the stem cell media, single cell division occurred after 48-72 $\mathrm{h}$, followed by the formation of large numbers of 'neurosphere-like' tumor spheres within 5-7 days. These tumor spheres were spherical or oval in suspending or semi-suspending states, with fine light refraction, consisting of 4-10 cells per sphere. Certain tumor spheres were of irregular morphology. The growth velocity was slow in the first few weeks, but within 2 weeks the majority of spheres had increased their diameters by 5-10-fold. Inspected under a microscope, the tumor spheres showed a smooth edge without prominent protrusion, and had poor light transmittance in the center as a result of a higher cell density. The majority of monolayer tumor cells still presented adherence, loss of proliferation, and subsequent differentiation, while tumor spheres remained suspended, continuing to proliferate and increase in cell numbers. Along with the growth of tumor spheres, a few adherent cells showed disaggregation, fragmentation, condensation and cell pyknosis, which manifested the progress of apoptosis. After approximately 2 weeks of culture, the formation of tumor spheres was observed and images were captured under a phase-contrast microscope (Fig. 1).

Proliferation and self-renewal assessment. In the proliferation assay, tumor spheres were dissociated into single-cell suspension and passaged at a ratio of 1:2 or 1:3. Cell cleavage occurred in $48 \mathrm{~h}$ and new tumor spheres formed within 1 week. Serial passage revealed the tumor sphere cells maintained a favorable proliferation ability after at least 4 generations. In a monoclonal formation assay, the single cells from tumor spheres were serially diluted and reseeded in microwells. Counted under a microscope, it demonstrated that more than $50 \%$ of the single cells in microwells were capable of forming new secondary tumor spheres, although the diameters were generally less than those of the primary spheres. Each new secondary sphere contained 10-40 cells, showing the same morphology as the parent sphere. This assay proved that individual cells from the parent tumor spheres were endowed with the ability to selfrenew and form new secondary sphere colonies.

Immunocytochemistry identification. The tumor spheres were immunostained using CD133, the committed BGSC marker (4,8-11). After firmly attaching to the anti-peeling slides, an 
immunocytochemical assay exhibited that the majority of tumor sphere cells were CD133-positive in a plasma membrane staining pattern under an immunofluorescence microscope. The nuclei were counterstained, exhibiting marked nuclear atypia. Images of the stained tumor spheres were captured, as shown in Fig. 2.

During induced differentiation, cell division in the spheres markedly decelerated and cells attached to the substrates, with cells migrating upon exposure to differentiation conditions. The tumor spheres became flat, exhibiting a radial morphology. The migrated cells formed monolayers, comprising various cell types with marked heteromorphism. Following differentiation in media with $15 \%$ FBS for 7 days, the cells were fixed and subjected to immunocytochemical detection. The results demonstrated that cells differentiated from the tumor spheres were positive for $\beta$-tubulin III and GFAP (Fig. 3), consistent with findings from other studies $(4,10,11)$. The results indicated that $\mathrm{CD}_{133^{+}}$tumor spheres were multipotent for at least two neural cell types, neurons and astrocytes, which shows a multilineage differentiation ability.

\section{Discussion}

Gliomas are the most frequently diagnosed and aggressive primary intracranial tumors, with poor outcome. The mortality rate has remained on the increase in recent years; only less than $5 \%$ of patients survive the first 5 years, even after radical therapeutic strategies (12). Despite advances in glioma research and treatment in recent decades, prognosis has not substantially improved and the median survival period has been elevated by only 9-10 weeks (13). Thus, it is necessary and practical to find new targets and investigate new therapies based on glioma pathogenetic mechanisms. The theory of BGSCs provided new targets for the cure of gliomas $(4-6,11,14)$. BGSCs are defined as a small population of cells capable of extensive proliferation, self-renewal, multipotent differentiation and tumor initiation. As the origins of glioma cells and the critical factors, BGSCs determine glioma propagation, progression and therapeutic resistance. Subsequently, BGSCs with a tumor-initiating ability have been isolated from GBMs, medulloblastomas, ependymomas and anaplastic oligoastrocytomas, and certain biological characteristics have been investigated $(4,5,8,11,15,16)$. These findings suggest a hierarchical model in which glioma arises from BGSCs and progresses through mechanisms similar to a developmental process. It is well known that the eradication of gliomas can only be accomplished by targeting BGSCs. Nevertheless, little is known with regards to the biological mechanisms of BGSCs.

CD133, a $120 \mathrm{kDa}$ cell-surface protein, which is a hallmark of normal human neural precursors, has been generally accepted as the marker of BGSCs regardless of whether they are solid tumors or glioma cell lines in vitro, although it is shared by other stem cells $(4,9-11,16)$. In addition, some investigators used a flow cytometry-based side population (SP) technique to isolate BGSCs based on the characteristic that BGSCs were capable of excluding the fluorescent dye Hoechst 33342 (17). However, Hoechst 33342 is a type of liposoluble DNA-binding fluorescent dye and is cytotoxic to various cell types. Therefore, it is inappropriate to compare the respective biological features

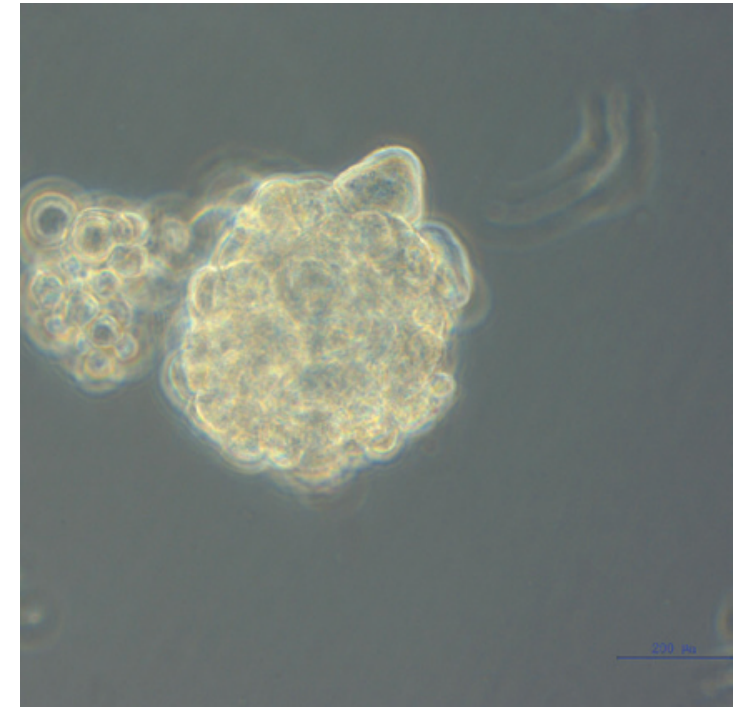

Figure 1. Tumor spheres cultured in serum-free stem cell medium (magnification, $\mathrm{x} 200$. Phase-contrast microscope).

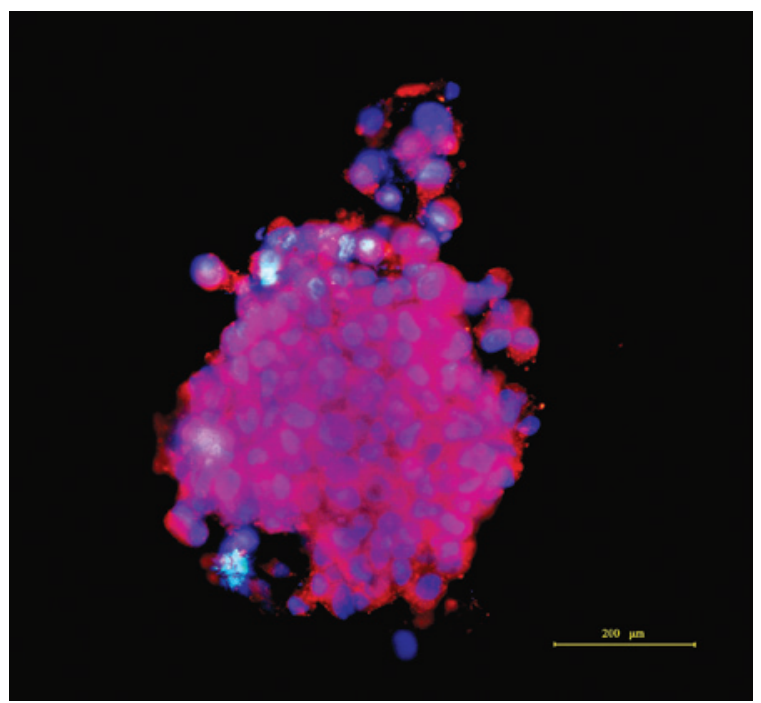

Figure 2. Tumor sphere cells were CD133-positive (magnification, x200). Cell nuclei were counterstained with DAPI (blue).

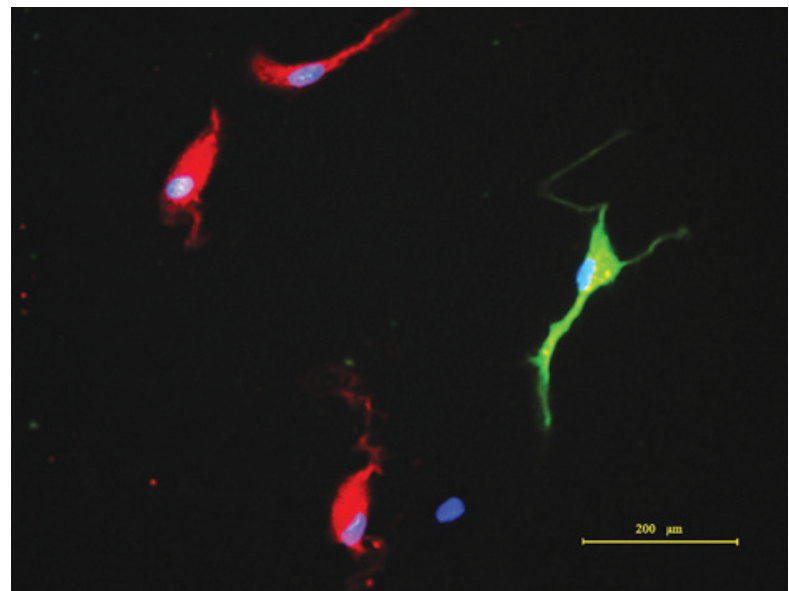

Figure 3. Tumor spheres differentiated to express the glial cell marker, GFAP (red) and neuronal marker, Tu-20 (green). Cell nuclei were counterstained with DAPI (blue) 
of sorted SP cells and non-SP cells. Thus, the SP sorting technique still calls for improvement. It is a key issue to find specific phenotype markers for BGSCs in subsequent studies, which may benefit further investigations on BGSCs.

The culture techniques for BGSCs are similar to the procedures for other stem cell types, such as serum-free media without adhesion factors and supplementation of rhEGF, rhFGF-b and B-27 (or N2 supplementation) (4-6,15,18,19). With regards to the sorting techniques, there are two basic methods available, one is immunomagnetic beads or fluorescent activated cell sorting (FACS) based on the surface marker CD133; and the other method is the SP cell sorting technique based on a drug-resistant gene, such as an ATP-binding cassette or a multidrug resistance gene (MDR) $(17,20)$. In either case, the supposed BGSCs are sorted, then identified and cultured. The procedures are complicated and expensive; following prolonged incubation, labeling, sorting and rinsing, the isolated BGSCs may not retain adequate viability for the subsequent culture process or relevant biological experiments. In this study, we simplified the steps of culture and identification to obtain expected BGSCs relatively rapidly and efficiently. The detailed culture process is the same as reported, but the isolation and identification are more convenient as described above. The results proved that the cultured tumor spheres could be serially passaged and possessed self-renewal ability, expressed the preferred BGSCs marker CD133, and differentiated into cells expressing $\beta$-tubulin III and GFAP, which were consistent with other findings $(4,6,17)$. These results proved the 'stemness' of tumor spheres, therefore they could be defined as CD133+ BGSCs.

Compared with conventional methods, we did not use the sorting techniques (immunomagnetic beads, FACS, or SP techniques) or animal experiments to initiate new glioma. As is known, there are mainly 2 types of methods to purify BGSCs; conditioned purification or specific marker sorting combined with conditioned culture. Although target cells can be sorted using specific markers, the limitations remain clear, such as the long duration of the procedure, the high expense, complicated steps and loss of cell viability. Since the BGSCs were cultured in suspended tumor spheres, only the suspension cells were harvested and serially passaged during culture. Thus, the majority of non-BGSCs were excluded after 3-5 generations and enough target cells of high purity were obtained. This result was confirmed by immunocytochemical staining, as shown in Fig. 2. The fibroblasts were also excluded during the suspension culture. When the BGSCs grew to a certain density and number, the propagation rate accelerated and the passage interval shortened, which were adapted for the relevant investigations. Furthermore, the animal experiment for tumorigenecity is currently regarded as a gold standard as it may verify the ability of BGSCs to initiate new gliomas, although the results are not always reliable. A number of experiments have found that either $\mathrm{CD}_{133^{+}}$or $\mathrm{CD}^{-33}{ }^{-}$glioma cells may result in gliomas $(9,19)$. For instance, investigators have reported that $\mathrm{CD} 133^{-}$glioma cells formed new gliomas in nude mice (19). Certain investigators $(21,22)$ also stated that it is not only the cancer stem cells that possess the ability of tumor initiation after a series of assays of lymphoma or leukemia stem cell xenografts. In our opinion, the NOD/SCID mice mostly selected for tumorigenecity experiments provide a glioma growing environment that is extremely different to the microenvironment of spontaneous gliomas. Subsequently, the animal experiments were not always appropriate for the identification of BGSCs and were not included in our study, which saved time and expense.

Currently, BGSCs have attracted much attention and have been extensively investigated in fundamental fields such as BGSC biological characteristics, and clinical fields such as immunotherapy or gene therapy targeting BGSCs. Cytological experiments are substantial for BGSC research and obtaining enough viable BGSCs for experiments is a pivotal problem. Although numerous types of glioma cell lines were employed for BGSCs experiments, their biological features have significantly changed following long-term culture and serial passage in vitro and cannot mirror the primary characteristics of gliomas. Therefore, culturing BGSCs from clinical samples is an appropriate option. Nevertheless, the classical procedures comprise relatively complicated steps and a series of assays, and BGSCs finally cultured and isolated may not retain sufficient viability and natural properties as expected. In this study, we simplified the procedures and successfully grew BGSCs from resected glioma samples of clinical cases. The flow cytometry or immunomagnetic bead sorting methods were not used; conditioned culture and serial passage were used instead. The animal experiments for glioma tumorigenecity were also not performed due to controversies regarding their effectiveness. Within 3-5 generations, a considerable number of BGSCs were harvested with a high purity, which was confirmed by immunocytochemical identification of the tumor spheres. The results proved that this method is time-saving, cost-effective and convenient, contributing to the subsequent studies on BGSCs.

\section{Acknowledgements}

This study was supported by the Foundation of Liaoning Provincial Commission of Science Technology (No. 2007408001-5).

\section{References}

1. Hess KR, Broglio KR and Bondy ML: Adult glioma incidence trends in the United States, 1977-2000. Cancer 101: 2293-2299, 2004.

2. Reardon DA, Rich JN, Friedman HS and Bigner DD: Recent advances in the treatment of malignant astrocytoma. J Clin Oncol 24: 1253-1265, 2006.

3. Jordan CT, Guzman ML and Noble M: Cancer stem cells. N Engl J Med 355: 1253-1261, 2006.

4. Galli R, Binda E, Orfanelli U, et al: Isolation and characterization of tumorigenic, stem-like neural precursors from human glioblastoma. Cancer Res 64: 7011-7021, 2004.

5. Hemmati HD, Nakano I, Lazareff JA, et al: Cancerous stem cells can arise from pediatric brain tumors. Proc Natl Acad Sci USA 100: 15178-15183, 2003

6. Singh SK, Clarke ID, Terasaki M, et al: Identification of a cancer stem cell in human brain tumors. Cancer Res 63: 5821-5828, 2003.

7. Tunici P, Irvin D, Liu G, et al: Brain tumor stem cells: new targets for clinical treatments? Neurosurg Focus 20: 27, 2006.

8. Yi L, Zhou ZH, Ping YF, et al: Isolation and characterization of stem cell-like precursor cells from primary human anaplastic oligoastrocytoma. Mod Pathol 20: 1061-1068, 2007.

9. Beier D, Hau P, Proescholdt M, et al: CD133(+) and CD133(-) glioblastoma-derived cancer stem cells show differential growth characteristics and molecular profiles. Cancer Res 67: 4010-4015, 2007. 
10. Huang Q, Dong J, Zhu YD, et al: [Isolation and culture of tumor stem cells from human brain glioma tissues]. Zhonghua Zhong Liu Za Zhi 28: 331-333, 2006.

11. Singh SK, Hawkins C, Clarke ID, et al: Identification of human brain tumour initiating cells. Nature 432: 396-401, 2004.

12. Claus EB and Black PM: Survival rates and patterns of care for patients diagnosed with supratentorial low-grade gliomas: data from the SEER program, 1973-2001. Cancer 106: 1358-1363, 2006.

13. Davis FG, Freels S, Grutsch J, Barlas S and Brem S: Survival rates in patients with primary malignant brain tumors stratified by patient age and tumor histological type: an analysis based on Surveillance, Epidemiology, and End Results (SEER) data, 1973-1991. J Neurosurg 88: 1-10, 1998.

14. Clarke MF, Dick JE, Dirks PB, et al: Cancer stem cellsperspectives on current status and future directions: AACR Workshop on cancer stem cells. Cancer Res 66: 9339-9344, 2006.

15. Taylor MD, Poppleton H, Fuller C, et al: Radial glia cells are candidate stem cells of ependymoma. Cancer Cell 8: 323-335, 2005.

16. Yuan X, Curtin J, Xiong Y, et al: Isolation of cancer stem cells from adult glioblastoma multiforme. Oncogene 23: 9392-9400, 2004.
17. Kondo T, Setoguchi $\mathrm{T}$ and Taga T: Persistence of a small subpopulation of cancer stem-like cells in the C6 glioma cell line. Proc Natl Acad Sci USA 101: 781-786, 2004.

18. Lee J, Kotliarova S, Kotliarov Y, et al: Tumor stem cells derived from glioblastomas cultured in bFGF and EGF more closely mirror the phenotype and genotype of primary tumors than do serum-cultured cell lines. Cancer Cell 9: 391-403, 2006.

19. Wang J, Sakariassen PO, Tsinkalovsky O, et al: CD133 negative glioma cells form tumors in nude rats and give rise to CD133 positive cells. Int J Cancer 122: 761-768, 2008.

20. Chua C, Zaiden N, Chong KH, et al: Characterization of a side population of astrocytoma cells in response to temozolomide. J Neurosurg 109: 856-866, 2008.

21. Kelly PN, Dakic A, Adams JM, Nutt SL and Strasser A: Tumor growth need not be driven by rare cancer stem cells. Science 317: 337, 2007.

22. Kennedy JA, Barabe F, Poeppl AG, Wang JC and Dick JE: Comment on 'Tumor growth need not be driven by rare cancer stem cells'. Science 318: 1722, 2007. 